\title{
Calibration method to assess the accuracy of measurements devices using the theory of uncertainty
}

\author{
O.M. Vasilevskyi ${ }^{\star}$ \\ Department of Metrology and industrial automation, Vinnytsya National Technical University, 95 Khmelnitskoye Shose, \\ 21021 Vinnitsya, Ukraine
}

Received: 5 July 2014 / Accepted: 2nd September 2014

\begin{abstract}
The development of evaluation methods for precision measurement and inter-verification interval measurement for measurement devices based on the concept of the uncertainty of measurement is needed in order to establish the timing of the next scheduled or unscheduled verification of the correspondence of metrological characteristics to their standardised values. The definition of the timing (periodicity) of the metrological verification of measurement devices as used in business or health care, while monitoring environmental conditions and proving health and safety at work, is an urgent issue in scientific research, upon which depends the quality of goods and services. A method of evaluating the accuracy of measurements and the inter-verification interval for measurement devices is proposed, based on using international standards guidelines for quality measurement. The calibration method for evaluating the inter-verification interval of measurement devices was tested during the metrological certification of the measurement device for the moment of inertia of electric motors.
\end{abstract}

Keywords: Metrological certification, inter-verification interval, measurement uncertainty, quality assurance of measurements

\section{Introduction}

In order to enable a report to be made on the results of measurement of physical properties, quantitative results must be submitted in such a way that their reliability may be adequately assessed. Without such values, the results of measurement cannot be compared either with each other or with the reference values submitted in the specifications or standards provided with measurement devices. Therefore, to ensure international consensus in measurement science, there have been developed easily carried-out, understandable and broadly accepted methods of processing the results of measurements - the concept of uncertainty in measurement [1-5].

It is accepted that measurement instruments are technical devices which are characterised by standardised metrological characteristics [2,5-7]. The reliability of measurement devices is determined by their ability to withstand the metrological parameters of regulated limits. A result obtained beyond these set boundaries is classified as a metrological failure. The correspondence of metrological characteristics to their standardised values is established during the course of verification (metrological control) or metrological certification of measurement devices. The introduction of the concept of measurement uncertainty to international standards for the evaluation and definition

^ Correspondence: wasilevskiy@mail.ru of the characteristics of precision measurements [1] and the evaluation of quality electrotechnical items [2] requires the development of methods for the assessment (establishment) of the inter-verification interval of the measurement device, the procedure of which should be based on the theory of uncertainty of measurement [4].

The development of evaluation methods for precision measurement and inter-verification interval measurement for measurement devices based on the concept of the uncertainty of measurement is needed in order to establish the timing of the next scheduled or unscheduled verification of the correspondence of metrological characteristics to their standardised values. The definition of the timing (periodicity) of the metrological verification of measurement devices as used in business or health care, while monitoring environmental conditions and proving health and safety at work, is an urgent issue in scientific research, upon which depends the quality of goods and services.

Given the above, the purpose of this article is to develop a method for evaluating the accuracy of performance measurement and the inter-verification interval for measurement devices based on the theory of the uncertainty of measurement, that will allow the timing to be established for scheduled verification of measurement devices, according to international standards that apply to the assessment of the quality of electrical products. In addition, the proposed evaluation method of measuring 
accuracy must be tested during the metrological certification of measurement device for the moment of inertia of electric motors.

\section{Analysis of the status of research and publications}

The existing academic resources quite satisfactorily consider separate theoretical approaches to evaluating and expressing uncertainty $[1-5,7]$ and the theoretical approaches to determine intermediate verification (interverification) intervals of measuring instruments based on the limits of metrological characteristics of instability. The leeway in metrological characteristics is based on the theory of measurement error and the reliability of means without regard to the concept of uncertainty of measurement $[6,8-10]$. Thus, to date, there is no approach to determining the inter-verification range of measuring instruments based on the theory of uncertainty of measurement. Therefore there is a need to develop mathematical tools to determine and define the inter-verification range of measuring instruments based on international standards for evaluation characteristics of measurement accuracy - the theory of uncertainty of measurement.

We know that if it is possible to determine, at least to an approximate degree, the average number of metrological failures $q$ in the general stream of rejections of measuring instruments, the estimated functional accuracy of measurements without measurement rejections $P_{M}(t)$ during the duration of operation $t[6,9]$ may be demonstrated by $[5,6]$ :

$$
P_{M}(t)=1-q(t)[1-P(t)],
$$

where $P(t)$ is the probability of failure-free operation of the measuring device (technical reliability) for the time of operation $t$.

If the average number of metrological failures $q(t)$ cannot be determined, then $P_{M}(t)=P(t)$ should be used.

We also know from the literature $[6,8-10]$ that key indicators that can be used to calculate the characteristics of metrological reliability are: the probability of failure-free operation; the frequency of metrological failures; mean time to first failure in metrology; the parameter flow of metrological failures; and time to first metrological failure.

However, in the information on the means of measurement that are submitted for testing to provide type approval or metrological certification of the means of measurement, there is often no reliable information about the instability of the metrological characteristics of the measurement means required for justifying the assignment of an initial inter-verification interval for the measurement instrument. In these cases, it is possible to estimate such intervals by using fixed values for the reliability parameters as specified in the technical specifications and documentation for the measurement devices, or by using analogue information on the inter-verification intervals, followed by correction of operational values on the basis of data on the frequency of usage and the measurement conditions.

\section{Theoretical approach to the definition of the inter-verification interval of measurement means based on the concept of the uncertainty of measurement}

To determine the inter-verification interval of a measurement device based on the concept of measurement uncertainty, a theoretical approach is proposed below.

Experimental evaluation of measurement uncertainty in the lower and upper measurement range of a measurement device through digitized gradations, a series of measurements must be conducted at the lower limits of measurement of the measuring device (minimum values standardised by measurement instruments) within the measurement range of the measuring device, and the upper limits of measurement of the measurement device (the maximum value that is specified in the technical documentation). At the same time the input of the measuring device must be sampled in the set of values of measurement signals that correspond to the specified measurement range in the technical documentation. Hence, experimental research into the gradated limits of measurements may be performed using the methodology of sample measurements, sample signals, sample devices or comparison methods. Based on the experimental data, the standard uncertainty of type A for the lower limit of measurement for measuring mid-range and upper limit of measurement may be determined using the equation as follows [1]:

$$
u_{A}\left(\bar{x}_{K}\right)=\sqrt{\frac{\sum_{i=1}^{n}\left(x_{i, K}-\bar{x}_{K}\right)^{2}}{(n-1) n}},
$$

where $x_{i, K}$ is the quantity of values obtained in the $K$ th group of observations according to the lower limit of measurement within the measurement range, and the upper limit of measurement; $K$ is the number of groups monitored in the gradated increments of range measurements; $\bar{x}_{K}$ is the mean value of each group of observations under investigation; $n$ is the number of measured values in the $k$ th group of observations $[1,4,5]$.

From the results obtained by formula (2) of the experimental standard uncertainties of type $\mathrm{A}$, the largest value is set by as a maximum of the standard uncertainty of type $\mathrm{A} u_{A \max }(\bar{x})$, which is then used to determine the inter-verification interval of the measuring instruments.

The next stage in determining the inter-verification interval of the measurement device is the evaluation of the standard uncertainty of type B, which is determined by available information about the discarded remainder of repeatable effects that in theory, together with a justified degree of certainty, can be manifested in the process of measurement. In doing so, one should rely on information derived from prior measurements, acceptable working conditions for the means the measurement, the physical properties of the measured value, technical documentation data for the measurement device or means of reference 
data $[4,5]$. After evaluating the theoretically possible components of standard uncertainties of type B, it is necessary to calculate the combined total standard uncertainty of type $\mathrm{B} u_{c B}$ based on the known forms of representation of combined uncertainty [4].

After calculating the total measurement uncertainty of type $\mathrm{B}$, it is necessary to calculate the total uncertainty of the measurement result based on the maximum standard uncertainty of type A. The equation for calculating the total uncertainty of the measurement result in the absence of correlation is [1]:

$$
u_{c}(y)=\sqrt{\sum_{i=1}^{N}\left(\frac{\partial f}{\partial x_{i}}\right)^{2} u^{2}\left(x_{i}\right)},
$$

where $\frac{\partial f}{\partial x_{i}}=c_{i}$ are the sensitivity coefficients for the equation of transformation of measurement; $u\left(x_{i}\right)$ is the standard uncertainty evaluated as type A where the maximum standard uncertainty of type $\mathrm{A}$ is $u_{A \max }(\bar{x})$ according to type B.

If we assume that the coefficient of sensitivity to uncertainty of type $\mathrm{A}$ is 1 , and the sensitivity coefficient standard of uncertainty as estimated as type B is included in the calculation of the total uncertainty of type B, then equation (3) to estimate the total uncertainty of the measurement result may be written as:

$$
u_{c}(y)=\sqrt{\left[u_{A \max }(\bar{x})\right]^{2}+\left[u_{c B}\right]^{2}} .
$$

If there is a correlation between the input variables, the equation for determining the total uncertainty of the measurement result will be:

$$
\begin{aligned}
& u_{c}(y)= \\
& \sqrt{\sum_{i=1}^{N} c_{i}^{2} u^{2}\left(x_{i}\right)+2 \sum_{i=1}^{N-1} \sum_{j=i+1}^{N} c_{i} c_{j} u\left(x_{i}\right) u\left(x_{j}\right) r\left(x_{i}, x_{j}\right)},
\end{aligned}
$$

where

$$
r\left(x_{i}, x_{j}\right)=\frac{\sum_{l=1}^{N_{i j}}\left(x_{i_{l}}-\bar{x}_{i}\right)\left(x_{j_{l}}-\bar{x}_{j}\right)}{\sqrt{\sum_{l=1}^{N_{i j}}\left(x_{i_{l}}-\bar{x}_{i}\right)^{2} \sum_{l=1}^{N_{i j}}\left(x_{j_{l}}-\bar{x}_{j}\right)^{2}}}
$$

the correlation coefficient.

After calculating the total uncertainty of the measurement result, it is necessary to define the expanded measurement of uncertainty, which is attributed to the measuring methods as a standardised value, and noted in the test report of the technical documentation for the measuring device. Expanded measurement uncertainty is obtained by multiplying the total uncertainty of the measurement result by the coverage coefficient [1]:

$$
U_{N}=k_{P} u_{c}(y)
$$

where $k_{P}$ is the coverage coefficient, which is determined by information on the confidence probability $P$, and the effective number of degrees of freedom $\nu_{\text {eff }}$.

The value of the confidence probability $P$ is usually stated in the specifications or technical manuals for a given means of measurement. If the technical documentation does not specify a confidence probability, it is determined either experimentally or determined a priori $[4,6]$.

The effective number of degrees of freedom is calculated by the Welch-Satterthwaite equation:

$$
\nu_{\text {eff }}=(n-1) \frac{u_{c}^{4}(y)}{\left[u_{A \max }(\bar{x})\right]^{4}} .
$$

On the basis of the confidence probability $P$ and the effective number of degrees of freedom $\nu_{\text {eff }}$ from the Student table, the coverage coefficient $k_{P}$ is determined.

In the case that the effective number of degrees of freedom is greater than $30, \nu_{\text {eff }}>30$, the coverage coefficient is assumed to be $k_{0.9}=1.64$ when probability $P=0.9$; $k_{0.95}=1.96$ when $P=0.95 ; k_{0.99}=2.58$ when $P=0.99$ and $k_{0.9973}=3$ when $P=0.9973$.

After setting the standardised value of the expanded uncertainty of measurement under standard conditions, or the total measurement uncertainty value, it is necessary to define an operational longevity $t$ for the use of the measurement instrument. This may be defined on the basis of information about the intensity of the exploitation of the measurement means (number of working hours of operation per day), and also by the mean time to failure of the device or the stated value for operational duration to first metrological failure.

Having assigned certain standard values for the metrological characteristics this device should be used or tested under real applied conditions that differ from laboratory benchmarking, such conditions under which the measuring device will actually be used.

After lengthy trials of the measurement device, the total and summary expanded uncertainty is again calculated, based on the environmental conditions of actual use on-site. In this case, the calculations take into account the working conditions of operation, using real values of ambient temperature and other conditions of measurement. Thus, we may calculate operational uncertainty values using formulas (2)-(7). As a result of these calculations, operational values may be obtained for expanded uncertainty of measurement for $U_{E}$ under operating conditions, which is then used to specify the inter-verification interval of measurement instruments.

Based on the values of uncertainty of type A, standardised and theoretically possible expanded measurement uncertainty and operational expanded uncertainty of the measurement under the assumption of symmetry of the distribution of uncertainty, the first assessment of the inter-verification interval of the measurement device $T_{1}$ may be calculated using values of uncertainty as follows:

$$
T_{1}=t \frac{\ln \left(\frac{U_{E}}{k_{2 P-1} u_{A \max }(\bar{x})}\right)}{\ln \left(\frac{U_{N}}{k_{P} u_{A \max }(\bar{x})}\right)},
$$


where $k_{2 P-1}$ is the coverage coefficient, corresponding confidence probability $2 P-1$, i.e. a probability value that corresponds to the probability of metrological serviceability of the measurement device at the time of the definition of the inter-verification interval of the measurement device; where $t$ is the operational longevity of the measurement device.

The coverage coefficient ratio $k_{2 P-1}$ is determined from the Student table based information about the confidence probability $2 P-1$ and the effective number of degrees of freedom $\nu_{\text {eff }}$.

The second evaluation of the inter-verification interval $T_{2}$ may be calculated by the formula:

$$
T_{2}=t \frac{U_{E}-k_{2 P-1} u_{A \max }(\bar{x})}{U_{N}-k_{P} u_{A \max }(\bar{x})} .
$$

Based on calculated values of inter-verification intervals $T_{1}$ and $T_{2}$, an inter-verification interval is determined for a measurement device, which is assumed to be equal to the minimum value between the values of $T_{1}$ and $T_{2}$, i.e.:

$$
T=\min \left[T_{1}, T_{2}\right]
$$

Thus, the proposed method of determining the interverification interval for the means of measurement permits the establishment or specification of an inter-verification interval based on the concept of the uncertainty of measurement. This method meets international requirements for evaluating the accuracy of measurements, adhering to international unity for measurement definitions and can be used in the metrological certification of measuring instruments.

To verify the proposed theoretical statements used in calibration by a graduated method of determining interverification intervals of a measuring means, based on the concept of uncertainty, we consider the calculation of the inter-verification interval by taking the example of the metrological certification of a measurement device for measuring the moment of inertia of electric motors.

\section{Results of testing the proposed evaluation method for the inter-verification interval during metrological certification of a device measuring the moment of inertia of electric motors}

The principle of operation and mathematical model of the device measuring the moment of inertia is described in references [11-13]. The equation of the conversion device of the moment of inertia of the electric motor is:

$$
J=\frac{P T_{0}}{2 \ln \left(\frac{M_{k} l \xi h^{3}}{e r^{4} C \Delta \delta}\right)} N_{J},
$$

where $N_{J}$ is the number of pulses to the input of the timer-counter microcontroller that is part of the device to measure the moment of inertia over a time period of free oscillations of the rotor of the electric motor; $M_{k}$ is the torque at slip $S=1$, which is described in the Kloss equations $[14,15] ; l$ is the length of the measuring lever; $\xi$ is the modulus of elasticity of the membrane of the effort sensor; $h$ is the thickness of the membrane of the effort sensor; $e=0.17 ; r$ is the radius of the membrane of the effort sensor; $C$ is the coefficient of rigidity of the membrane of the effort sensor; $\Delta \delta$ is the standardised value of the absolute deviation of the measuring transducer; $J$ is the moment of inertia as measured; $P$ is the damping coefficient; $T_{0}$ is the duration of sample pulses, which fills the measuring period of the moment of inertia after the completion of the transition process (when electric motor power is cut and torque is diminishing as $M_{k}$ approaches zero) [13].

The essence of the method of measurement is the production of torque from the time that an electric motor is connected to a power supply, which is described by the Kloss formulae $[14,15]$. This production of torque through the measuring lever that is on one side attached to the rotor of the electric motor, and on the other side through the effort sensor, which is an elastic element. As later the electric motor is disconnected from the power supply, the resulting torque $M_{k}$ is reduced to zero during the time interval $T_{0}$. As the rotor of the electric motor undergoes free damped oscillations whose duration is due to the value of the moment of inertia of the rotor $J$, and the rigidity $C$ of the effort sensor, then measuring the magnitude of the torque and the time interval from the moment of powerdown to zero and knowing the value of $C$ of the rigidity of the effort sensor, it becomes possible to determine the value of the moment of inertia of the electric motor (11).

So, for the metrological certification of the measurement device to determine the moment of inertia of electric motors, we need to set a fixed torque value $M_{k}$ and then in the self-braking mode of operation of the electric motor perform measurements of the moment of inertia. The sample moment $M_{\text {exe }}$ is suggested as being that created by using a sample set of weights. The equation to define the procedure for production of the sample torque is:

$$
M_{\text {exe }}=g R m_{\text {exe }}
$$

where $g$ is the rate of acceleration of gravity, which corresponds to $9.8066 \mathrm{~m} \mathrm{~s}^{-2} ; R$ is the radius of the disk $(9.9889 \mathrm{~cm})$, secured to the shaft of an electric motor, which is part of the torque measurement transducer; $m_{\text {exe }}$ is the mass of standard weights.

For calibration of a device to measure the moment of inertia, an asynchronous electric motor type AIR56A4 was used, having a nominal value of the moment of inertia of $J_{1}=0.007 \mathrm{~N} \mathrm{~m}^{2}$. To produce torque with slip $S=1$, it is necessary to create on the shaft of the electric motor a sample torque that corresponds to $M_{k}=0.85 \mathrm{~N} \mathrm{~m}$. To produce torque on a disk of radius $R$ which is fixed to the shaft of the electric motor via string length $l$, the value of the sample mass should equal $m_{\text {exe }}=867.726 \mathrm{~g}$.

From the results of previous measurements of the radius of the disk, it is known that the standard uncertainty of measurement of the radius of the disk is 
Table 1. Results of measurements of moments of inertia.

\begin{tabular}{cccccc}
\hline No. & $\begin{array}{c}\text { Value of } \\
\text { the moment } \\
\text { of inertia } \\
J \times 10^{-3}, \mathrm{~N} \mathrm{~m}^{2}\end{array}$ & No. & $\begin{array}{c}\text { Value of } \\
\text { the moment } \\
\text { of inertia } \\
J \times 10^{-3}, \mathrm{~N} \mathrm{~m}^{2}\end{array}$ & No. & $\begin{array}{c}\text { Value of } \\
\text { the moment } \\
\text { of inertia } \\
J \times 10^{-3}, \mathrm{~N} \mathrm{~m}^{2}\end{array}$ \\
\hline 1 & 7.132 & 8 & 7.128 & 15 & 7.175 \\
2 & 7.197 & 9 & 6.855 & 16 & 6.878 \\
3 & 6.805 & 10 & 7.153 & 17 & 7.191 \\
4 & 7.157 & 11 & 7.192 & 18 & 6.823 \\
5 & 6.952 & 12 & 6.863 & 19 & 7.147 \\
6 & 7.134 & 13 & 7.176 & 20 & 6.792 \\
7 & 7.171 & 14 & 6.883 & 21 & 7.165 \\
\hline
\end{tabular}

$u_{\mathrm{Ar}}=1.04 \times 10^{-3} \mathrm{~mm}$, and with the technical specification data on the sample means of measuring mass with a maximum load of $1000 \mathrm{gm}$, it is known that the mass of the weights may be measured with an absolute deviation in measurement of $\Delta_{m}=1 \mathrm{mg}$. The uncertainty of mass measurement if it is assumed that the absolute deviation is distributed evenly may be calculated by:

$$
u_{B m}=\frac{\Delta_{m}}{\sqrt{3}}=\frac{10^{-3} \mathrm{~g}}{1.73}=0.58 \times 10^{-3} \mathrm{~g} .
$$

Thus, the total uncertainty in producing torque may be calculated by the formula:

$$
u_{c}\left(M_{\text {exe }}\right)=\sqrt{\left(\frac{\partial M_{\text {exe }}}{\partial R}\right)^{2} u_{\mathrm{Ar}}^{2}+\left(\frac{\partial M_{\text {exe }}}{\partial m}\right)^{2} u_{B m}^{2}}
$$

where

$$
\frac{\partial M_{\text {exe }}}{\partial R}=g m_{\text {exe }}=8.509 \mathrm{~m} \mathrm{~s}^{-2} \mathrm{~kg}
$$

is the sensitivity coefficient dependent on the disk radius;

$$
\frac{\partial M_{\text {exe }}}{\partial m_{\text {exe }}}=g R=0.9796 \mathrm{~m}^{2} \mathrm{~s}^{-2}
$$

is the sensitivity coefficient dependent on the mass of a sample weight.

Substituting the calculated sensitivity coefficients and uncertainty in the formula (14), we obtain the value of the total uncertainty of the production of the sample torque as $u_{c 1}\left(M_{\text {exe }}\right)=8.87 \times 10^{-6} \mathrm{~N} \mathrm{~m}$.

After setting the sample torque in the manner described above, a series of measurements of the moments of inertia is performed by using a device for the measurement of the moment inertia of electric motors. The results of measurements of moments of inertia are listed in Table 1.

Based on the results of measurements of moments of inertia (Tab. 1), we may calculate the standard uncertainty of type A by equation (2). Substituting the results of measurements in equation (2), we obtain a value for a standard experimental uncertainty of measurement by:

$$
\begin{aligned}
u_{A}\left(\bar{J}_{1}\right) & =\sqrt{\frac{\sum_{i=1}^{n}\left(J_{i, 1}-\bar{J}_{1}\right)^{2}}{n(n-1)}} \\
& =\sqrt{\frac{\sum_{i=1}^{21}\left(J_{i, 1}-7.046 \times 10^{-3}\right)^{2}}{21 \times(21-1)}} \\
& =34.16 \times 10^{-6} \mathrm{~N} \mathrm{~m}^{2} .
\end{aligned}
$$

For calibration of a device to measure the moment of inertia in a different range, an asynchronous electric motor type AIR80A2 was used, having a nominal value of the moment of inertia of $J_{2}=0.015 \mathrm{~N} \mathrm{~m}^{2}$. To produce torque with slip $S$ close to 1, the electric motor shaft must create an exemplary torque that corresponds to $M_{k}=5 \mathrm{~N} \mathrm{~m}$. To produce torque on a disk of radius $R$ which is fixed to the shaft of the electric motor via string length 1 , the value of the sample mass should equal $m_{e x e}=5104.273 \mathrm{~g}$.

The total uncertainty in the production of a sample value of torque, which arises due to residual nonincorporated systematic effects related to the limited accuracy of measurement instruments of mass and the radius of the disk, is as according to formula (14):

$$
\begin{aligned}
\frac{\partial M_{\text {exe }}}{\partial R} & =g m_{\text {exe }}=50.056 \mathrm{~m} \mathrm{~s}^{-2} \mathrm{~kg} ; \\
\frac{\partial M_{\text {exe }}}{\partial m_{\text {exe }}} & =g R=0.9796 \mathrm{~m}^{2} \mathrm{~s}^{-2} ; \\
u_{c 2}\left(M_{\text {exe }}\right) & =5.21 \times 10^{-5} \mathrm{~N} \mathrm{~m} .
\end{aligned}
$$

Having assigned sample values of the moments of inertia, a series of measurements was made that is listed in Table 2.

Substituting the experimental data as listed in Table 2, we may calculate the standard uncertainty of type A of the measurement of the moment of inertia of an electric 
Table 2. Results of measurements of moments of inertia.

\begin{tabular}{|c|c|c|c|c|c|}
\hline No. & $\begin{array}{c}\text { Value of } \\
\text { the moment } \\
\text { of inertia } \\
J \times 10^{-3}, \mathrm{~N} \mathrm{~m}^{2}\end{array}$ & No. & $\begin{array}{c}\text { Value of } \\
\text { the moment } \\
\text { of inertia } \\
J \times 10^{-3}, \mathrm{~N} \mathrm{~m}^{2}\end{array}$ & No. & $\begin{array}{c}\text { Value of } \\
\text { the moment } \\
\text { of inertia } \\
J \times 10^{-3}, \mathrm{~N} \mathrm{~m}^{2}\end{array}$ \\
\hline 1 & 15.121 & 8 & 15.315 & 15 & 15.205 \\
\hline 2 & 15.017 & 9 & 15.122 & 16 & 15.012 \\
\hline 3 & 14.89 & 10 & 15.112 & 17 & 15.323 \\
\hline 4 & 15.116 & 11 & 15.015 & 18 & 15.303 \\
\hline 5 & 15.102 & 12 & 15.313 & 19 & 14.97 \\
\hline 6 & 15.087 & 13 & 15.018 & 20 & 15.114 \\
\hline 7 & 15.211 & 14 & 14.91 & 21 & 15.196 \\
\hline
\end{tabular}

Table 3. Results of measurements of moments of inertia.

\begin{tabular}{|c|c|c|c|c|c|}
\hline No. & $\begin{array}{c}\text { Value of } \\
\text { the moment } \\
\text { of inertia } \\
J \times 10^{-3}, \mathrm{~N} \mathrm{~m}^{2}\end{array}$ & No. & $\begin{array}{c}\text { Value of } \\
\text { the moment } \\
\text { of inertia } \\
J \times 10^{-3}, \mathrm{~N} \mathrm{~m}^{2}\end{array}$ & No. & $\begin{array}{c}\text { Value of } \\
\text { the moment } \\
\text { of inertia } \\
J \times 10^{-3}, \mathrm{~N} \mathrm{~m}^{2}\end{array}$ \\
\hline 1 & 4.178 & 8 & 4.365 & 15 & 4.011 \\
\hline 2 & 4.388 & 9 & 4.085 & 16 & 4.391 \\
\hline 3 & 4.289 & 10 & 4.015 & 17 & 4.039 \\
\hline 4 & 4.394 & 11 & 4.011 & 18 & 4.383 \\
\hline 5 & 4.286 & 12 & 4.355 & 19 & 4.397 \\
\hline 6 & 4.378 & 13 & 4.054 & 20 & 4.289 \\
\hline 7 & 4.386 & 14 & 4.016 & 21 & 4.036 \\
\hline
\end{tabular}

motor (calibration uncertainty) by formula (2):

$$
\begin{aligned}
u_{A}\left(\bar{J}_{2}\right) & =\sqrt{\frac{\sum_{i=1}^{n}\left(J_{i, 2}-\bar{J}_{2}\right)^{2}}{n(n-1)}} \\
& =\sqrt{\frac{\sum_{i=1}^{21}\left(J_{i, 2}-15.118 \times 10^{-3}\right)^{2}}{21 \times(21-1)}} \\
& =28.36 \times 10^{-6} \mathrm{~N} \mathrm{~m}^{2} .
\end{aligned}
$$

For calibration of a device to measure the moment of inertia $J_{3}=0.0042 \mathrm{~N} \mathrm{~m}^{2}$, an asynchronous electric motor type AIR56A2 was used. For this type of electric motor to produce slip $S$ close to 1 , the electric motor must create an exemplary torque that corresponds to $M_{k}=0.64 \mathrm{~N} \mathrm{~m}$. To produce torque on a disk of radius $R$ which is fixed to the shaft of the electric motor, the value of the sample mass should equal $m_{\text {exe }}=653.346 \mathrm{~g}$.

The total uncertainty in the production of a sample value of torque, which arises due to residual nonincorporated systematic effects related to the limited accuracy of measurement instruments of mass and the radius of the disk, is as according to the formula (14):

$$
\begin{aligned}
\frac{\partial M_{\text {exe }}}{\partial R} & =g m_{\text {exe }}=6.407 \mathrm{~m} \mathrm{~s}^{2} \mathrm{~kg} ; \\
\frac{\partial M_{\text {exe }}}{\partial m_{\text {exe }}} & =g R=0.9796 \mathrm{~m}^{2} \mathrm{~s}^{2} \\
u_{c 3}\left(M_{\text {exe }}\right) & =6.69 \times 10^{-6} \mathrm{~N} \mathrm{~m} .
\end{aligned}
$$

The results of measuring the moment of inertia are presented in Table 3.
Substituting the experimental data from Table 3 in equation (2), we obtain the standard uncertainty of type A measuring moment of inertia of the electric motor (calibration uncertainty), that is:

$$
\begin{aligned}
u_{A}\left(\bar{J}_{3}\right) & =\sqrt{\frac{\sum_{i=1}^{n}\left(J_{i, 3}-\bar{J}_{3}\right)^{2}}{n(n-1)}} \\
& =\sqrt{\frac{\sum_{i=1}^{21}\left(J_{i, 3}-4.226 \times 10^{-3}\right)^{2}}{21 \times(21-1)}} \\
& =35.72 \times 10^{-6} \mathrm{~N} \mathrm{~m}^{2} .
\end{aligned}
$$

The formula for determining the inter-verification interval of a measurement device incorporates the highest value, maximum, experimental standard uncertainty of type A. From the conducting of experimental research, it may be shown that standard uncertainty of type A may reach its maximum value when the smallest moment of inertia is measured with the value of $\bar{J}_{3}=0.0042 \mathrm{~N} \mathrm{~m}^{2}$. Therefore, to assess the inter-verification interval of a measurement device in subsequent calculations, we may use the maximum uncertainty of measurement of a moment of inertia of type A equal to $u_{A \max }\left(\bar{J}_{3}\right)=35.72 \times 10^{-6} \mathrm{~N} \mathrm{~m}^{2}$.

To calculate the total and expanded uncertainty of measurement, we should perform the evaluation of components with uncertainty of type B, which are manifested by non-incorporated residual systematic effects and limited properties of the constituent elements of a measurement means for the moment of inertia. 
In as much as the constituent elements of the moment of inertia of the measurement device includes an effort sensor, then we may estimate the uncertainty of type B, which is due to the existence of a consolidated error $\gamma=$ $0.15 \%$ when the maximum effort $Q=20 \mathrm{~kg}$, assuming a uniform law of error distribution by the formula:

$$
\begin{aligned}
u_{B, s} & =\frac{\gamma Q}{\sqrt{12} \times 100 \%}=0.15 \% \times \frac{20}{3.46 \times 100 \%} \\
& =8.67 \times 10^{-3} \mathrm{~kg} .
\end{aligned}
$$

Uncertainty of type B, which is caused by the presence of non-incorporated systematic effects associated with the presence of errors in the length of the measurement shaft which do not exceed $\Delta_{l}= \pm 0.01 \times 10^{-3} \mathrm{~m}$, may be shown by:

$$
u_{B, l}=\frac{\Delta_{l}}{\sqrt{12}}=\frac{ \pm 0.01}{\sqrt{3}}=5.77 \times 10^{-6} \mathrm{~m} .
$$

Let us place a value for the component of uncertainty due to the change in frequency of the quartz resonator during the formation period of sample pulses, when the frequency of the quartz resonator may be described by $f_{0}=20 \mathrm{MGz}$, $T_{0}=1 / f_{0}=0.05 \times 10^{-6} \mathrm{~s}$ which fills the measurement interval of the moment of inertia, given a temperature deviation in the ambient air temperature where $t_{v}=25^{\circ} \mathrm{C}$, from a normal temperature where $t_{n}=20{ }^{\circ} \mathrm{C}$, through the temperature coefficient of frequency changes of the quartz resonator where $k_{t}= \pm 1.5 \times 10^{-6} /{ }^{\circ} \mathrm{C}$ as specified in the technical documentation. This demonstrated by the equation:

$$
\begin{aligned}
u_{B, t} & =T_{0} \frac{\left|t_{v}-t_{n}\right|}{\sqrt{3}} k_{t} \\
& =0.05 \times 10^{-6} \times \frac{(25-20)}{1.73} \times 1.5 \times 10^{-6} \\
& =0.22 \times 10^{-12} \mathrm{~s} .
\end{aligned}
$$

The uncertainty caused by the presence of a certain response time by the analogue microcontroller comparator to the appearance of an input signal according to the specifications for the microcontroller does not exceed $\Delta t=0.5 \times 10^{-6} \mathrm{~s}$, as calculated by the formula:

$$
u_{B, \Delta t}=\frac{\Delta t}{\sqrt{12}}=\frac{0.5 \times 10^{-6}}{3.46}=0.14 \times 10^{-6} \mathrm{~s} .
$$

The total standard uncertainty of type B with regard to the transformation equation (11) above and the estimated components of uncertainty of type B (14), (18)-(21), enables us to find the positive square root of the total variance of type B, which is described by the formula:

$$
\begin{aligned}
u_{B c}^{2}= & \left(\frac{\partial J}{\partial M_{k}}\right)^{2}\left[u_{c 2}\left(M_{e x e}\right)\right]^{2}+\left(\frac{\partial J}{\partial \Delta \delta}\right)^{2} u_{B, s}^{2} \\
& +\left(\frac{\partial J}{\partial l}\right)^{2} u_{B, l}^{2}+\left(\frac{\partial J}{\partial T_{0}}\right)^{2}\left[u_{B, t}^{2}+u_{B, \Delta t}^{2}\right]
\end{aligned}
$$

where

$$
\begin{gathered}
\frac{\partial J}{\partial M_{k}}=-\frac{0.5 M_{k}^{-1} P T_{0} N_{J}}{\ln \left(\left[\frac{M_{k} l \xi h^{3}}{e r^{4} C \Delta \delta}\right]^{2}\right)}=-39.47 \times 10^{-6} \mathrm{~m} \\
\frac{\partial J}{\partial \Delta \delta}=\frac{0.5 \Delta \delta^{-1} P T_{0} N_{J}}{\ln \left(\left[\frac{M_{k} l \xi h^{3}}{e r^{4} C \Delta \delta}\right]^{2}\right)}=13.22 \times 10^{-3} \mathrm{~N} \mathrm{~m}^{2} \mathrm{~kg}^{-1} \\
\frac{\partial J}{\partial l}=-\frac{0.5 l^{-1} P T_{0} N_{J}}{\ln \left(\left[\frac{M_{k} l \xi h^{3}}{e r^{4} C \Delta \delta}\right]^{2}\right)}=-2.64 \times 10^{-3} \mathrm{~N} \mathrm{~m} \\
\frac{\partial J}{\partial T_{0}}=\frac{P N_{J}}{2 \ln \left(\left[\frac{M_{k} l \xi h^{3}}{e r^{4} C \Delta \delta}\right]^{2}\right)}=7.93 \times 10^{3} \mathrm{~N} \mathrm{~m} \mathrm{~s}^{-1}
\end{gathered}
$$

the sensitivity coefficients are for the torque, for sensor error effects, the length of the measuring shaft, the period of sample pulses, respectively.

Substituting the calculated values of sensitivity coefficients and standard uncertainties of type B in equation (22), we obtain the value of the total standard uncertainty of type $\mathrm{B}$, which is $u_{B c}=11.16 \times 10^{-4} \mathrm{~N} \mathrm{~m}^{2}$.

The total uncertainty of the measurement result for the moment of inertia with regard to the maximum experimental uncertainty of type A (17) and the total uncertainty of type B (22) may be calculated by the formula:

$$
u_{c}=\sqrt{u_{A}^{2}\left(\bar{J}_{3}\right)+u_{B c}^{2}}=11.17 \times 10^{-4} \mathrm{~N} \mathrm{~m}^{2} .
$$

To calculate the expanded uncertainty as defined in technical documentation for a device measuring the moment of inertia, we should calculate the effective number of degrees of freedom as follows:

$$
\begin{aligned}
\nu_{e f f} & =\frac{u_{c}^{4}}{\sum_{i=1}^{N} \frac{u_{i}^{4}}{\nu_{i}}}=(n-1) \frac{u_{c}^{4}}{u_{A}^{4}\left(\bar{J}_{3}\right)} \\
& =20 \times \frac{\left(11.17 \times 10^{-4}\right)^{4}}{\left(35.72 \times 10^{-6}\right)^{4}}=19 \times 10^{6} .
\end{aligned}
$$

Next, using the Student table, let us define the coefficient of coverage $k_{P}$ using the values of the effective number of degrees of freedom (24) and confidence probability, which is assumed to be $P=0.95$ based on information about the analogue probabilities. This will be $k_{P}=1.96$.

Knowing the coverage coefficient and the total uncertainty of the measurement result of the moment of inertia, we obtain an expanded uncertainty of measurement that is specified in the technical documentation for a measurement device for the moment of inertia of an electric motor, that is:

$$
U_{N}=k_{P} u_{c}=1.96 \times 11.17 \times 10^{-4}=2.19 \times 10^{-3} \mathrm{~N} \mathrm{~m}^{2} \text {. }
$$

Next, assuming that the intensity of operation of the measurement device is $7 \mathrm{~h}$ per day, and setting the experimentally determined time to first failure, which for the measurement device that determines the moment of inertia for 
the electric motors is $3500 \mathrm{~h}$, we define a calendar service life of $t$, which equates to some 2 calendar years.

After prolonged use under real conditions using the measurement device, we may now calculate the components of uncertainty of type B.

These type B uncertainties result from:

- the presence of the consolidated errors of the effort sensor, as calculated by (18) and being $u_{B, s}=8.67 \times$ $10^{-3} \mathrm{~kg}$;

- the presence of the non-consolidated systematic effects related to the limits of the ability to measure the measuring lever, as calculated by (19) and being equal to $u_{B, l}=5.77 \times 10^{-6} \mathrm{~m}$;

- the presence of the response time of the analogue microcontroller comparator to the appearance of the input signal is calculated by formula $(21)$ and is equal to the same value calculated by $u_{B, \Delta t}=0.14 \times 10^{-6} \mathrm{~s}$;

- the deviation of the ambient temperature of the environment during testing when $t_{v 2}=18{ }^{\circ} \mathrm{C}$ from the temperature under normal conditions when $t_{n}=$ $20{ }^{\circ} \mathrm{C}$, enables us to calculate through the temperature coefficient the change of frequency of the quartz resonator $\left(k_{t}= \pm 1.5 \times 10^{-6} /{ }^{\circ} \mathrm{C}\right)$ by the formula:

$$
\begin{aligned}
u_{B, t 2} & =T_{0} \frac{\left|t_{v 2}-t_{n}\right|}{\sqrt{3}} k_{t} \\
& =0.05 \times 10^{-6} \times \frac{|18-20|}{1.73} 1.5 \times 10^{-6} \\
& =8.67 \times 10^{-14} \mathrm{~s} .
\end{aligned}
$$

The value of combined measurement uncertainty of type $\mathrm{B}, u_{B c e}$, may be calculated by formula (23) taking into account the calculated uncertainties of measurement of type $\mathrm{B}$ after the testing of the measurement device for the moment of inertia in actual use. As a result, we may obtain the value of the combined standard uncertainty of type $\mathrm{B}$, which is $u_{B c e}=11.16 \times 10^{-4} \mathrm{~N} \mathrm{~m}^{2}$.

Because the value of the combined measurement uncertainty of type B remains unchanged, then the value of the combined uncertainty of the measurement resulting from real conditions remains as $u_{c e}=11.17 \times 10^{-4} \mathrm{~N} \mathrm{~m}^{2}$.

The effective number of degrees of freedom is determined by (24) and is also equal to the previous value $\nu_{\text {eff }}=19 \times 10^{6}$.

The coefficient of coverage $k_{2 P-1}$, which corresponds to the confidence probability $2 P-1$, i.e. the probability value that corresponds to the probability of metrological serviceability of the measuring device under real conditions where the technical reliability of the measuring device is $P=0.95$, and the probability of metrological serviceability is thus $2 P-1=2 \times 0.95-1=0.9$, may be determined from the Student table for the effective number of degrees of freedom where $\nu_{\text {eff }}>30$ and the probability of metrological serviceability measuring device under actual conditions is $k_{2 P-1}=1.64$.

When the coverage coefficient is $k_{2 P-1}=1.64$, the value of the extended uncertainty $U_{E}$ under real condi- tions of the operation of the measuring device is:

$$
\begin{aligned}
U_{E} & =k_{2 P-1} u_{c e}=1.64 \times 11.17 \times 10^{-4} \\
& =1.83 \times 10^{-3} \mathrm{~N} \mathrm{~m}^{2} .
\end{aligned}
$$

Based on the values of the standardised expanded uncertainty that is theoretically possible, the expanded uncertainty under real conditions $U_{E}$ (assuming a probability of metrological serviceability $2 P-1$ for the measurement device) and the maximum measurement uncertainty of type A $u_{A}\left(\bar{J}_{3}\right)$, let us calculate the initial assessment of the inter-verification interval $T_{1}$ of the measurement device for the moment of inertia of electric motors by formula (8), based on experimental period time to first failure $t=2$ years. The value of the first inter-verification interval corresponds to:

$$
\begin{aligned}
T_{1} & =t \frac{\ln \left(\frac{U_{E}}{k_{2 P-1} u_{A \max }\left(\bar{J}_{3}\right)}\right)}{\ln \left(\frac{U_{\dot{I}}}{k_{P} u_{A \max }\left(\bar{J}_{3}\right)}\right)} \\
& =2 \frac{\ln \left(\frac{1.83 \times 10^{-3}}{1.64 \times 35.72 \times 10^{-6}}\right)}{\ln \left(\frac{2.19 \times 10^{-3}}{1.96 \times 35.72 \times 10^{-6}}\right)} \approx 1.99 \text { years. }
\end{aligned}
$$

The second value of the inter-verification interval for a measurement device for the moment of inertia of electric motors $T_{2}$, is calculated by formula (9), and is:

$$
\begin{aligned}
T_{2} & =t \frac{U_{E}-k_{2 P-1} u_{A \max }\left(\bar{J}_{3}\right)}{U_{\dot{I}}-k_{P} u_{A \max }\left(\bar{J}_{3}\right)} \\
& =2 \frac{1.83 \times 10^{-3}-1.64 \times 35.72 \times 10^{-6}}{2.19 \times 10^{-3}-1.96 \times 35.72 \times 10^{-6}}=1.67 \text { years. }
\end{aligned}
$$

Thus, the inter-verification interval for a measurement device for the moment of inertia of electric motors according to formula (10) may be assumed to be equal to the minimum values between $T_{1}$ and $T_{2}$ :

$$
\begin{aligned}
T & =\min \left[T_{1}, T_{2}\right]=\min [1.99,1.67] \\
& =1.67 \text { years }=20 \text { months. }
\end{aligned}
$$

The value of the inter-verification interval in months may best be chosen from a row of natural numbers: $0.25 ; 0.5$; 1 and $2 ; 3 ; 4 ; 5 ; 6 ; 7 ; 8 ; 9 ; 10 ; 11 ; 12 ; 15,18 ; 21 ; 24 ; 30$ and so on at 6 -month intervals.

So, as a result of the metrological certification of the measurement device for the moment of inertia of electric motors, the accuracy of the measurements of the moment of inertia is based on the concept of uncertainty of measurement, the characteristics of which include the standard value of expanded uncertainty, which is $2.19 \times 10^{-3} \mathrm{~N} \mathrm{~m}^{2}$ with probability $P=0.95$ and the inter-verification interval of the measuring device which equals 20 months. 


\section{Conclusion}

The calibration method developed for inter-verification interval assessment of measurement devices allows standardised metrological characteristics of measurement devices to be set or refined and the timing of subsequent verifications may then be based on international requirements for the performance evaluation of the accuracy of measurement using the concept of uncertainty. This method allows for international consensus in measurements for the assessment of inter-verification intervals of measurement devices. Testing the calibration method of evaluating the accuracy of measurements and interverification intervals, made during the metrological certification of measuring devices for the moment of inertia of electric motors, demonstrates its validity and effectiveness.

\section{References}

1. ISO/IEC Guide 98-1:2009, Uncertainty of measurement Part 1: Introduction to the expression of uncertainty in measurement (Geneva, Switzerland, ISO, 2009), p. 32

2. IEC GUIDE 115-2007, Application of uncertainty of measurement to conformity assessment activities in the electrotechnical sector (Geneva, Switzerland, IEC, 2007), p. 54

3. Evaluation of measurement data. An introduction to the Guide to the expression of uncertainty in measurement and related documents, JCGM 104 (2009)

4. Evaluation of measurement data. Guide to the expression of uncertainty in measurement, JCGM 100, GUM 1995 with minor corrections, 1st edn. (2008)

5. K. Birch, Estimating Uncertainties in Testing, An Intermediate Guide to Estimating and Reporting, Uncertainty of Measurement in Testing (Measurement Good Practice Guide $\mathrm{N}^{\circ} 36$, British Measurement and Testing Association, 2001), p. 36, ISSN 1368-6550
6. O.M. Vasilevskyi, Rationing of metrological reliability parameters (Visnik Vinnitskogo politekhnichnogo institutu, Ukraine, 2011), Vol. 4, pp. 9-13, http://visnyk.vntu. edu.ua/article/view/1624

7. M. Dorozhovets, Z. Warsza, Evaluation of the uncertainty type A of autocorrelated measurement observations. Measurement Automation and Monitoring 2, 20-24 (2007)

8. Yu.S. Sysoev, Analysis of the drift of the metrological characteristics of measuring devices by means of Markov chains. Meas. Tech. 1, 19-24 (2012)

9. Yu.S. Sysoev, Analysis of the duration of metrologically reliable operation of measuring instruments by stochastic extrapolation techniques. Meas. Tech. 1, 8-15 (2011)

10. Yu.S. Sysoev, A.I. Tikhomirova, Analysis of the operational stability of measurement instruments by stochastic prediction of drift in their metrological characteristics. Meas. Tech. 6, 613-621 (2012)

11. O.M. Vasilevskyi, Advanced mathematical model of measuring the starting torque motors (Tekhnichna elektrodinamika, Ukraine, 2013), Vol. 6, pp. 76-81, http://techned.org . ua/eng/index .php?option=com _ content \\&view=article \\&id=595\\&Itemid=77

12. O.M. Vasilevskyi, A.V. Podzharenko, Evaluation of the measurement uncertainty of moment of inertia of the rotor in the amplitude of torsional vibrations (Visnyk Vinnytskogo politekhnichnogo instytutu, Ukraine), Vol. 4, pp. 5-9. http://visnyk. vntu.edu.ua/article/view/754

13. O.M. Vasilevskyi, Researches of metrology descriptions of mean measuring of moment inertia rotors electromotors. Proceedings of National Aviation University (2013), Vol. 2, pp. 62-67 (in Ukraine) http://jrnl.nau.edu.ua/index. php/visnik/article/view/5416

14. V. Subrahmanyan, Electric Drives (McGraw-Hill, USA, 1996), p. 715

15. R.M. Crowder, Electric Drives and their Controls (Oxford Unoversity Press, Oxford, 1998), p. 256 